Supporting Materials for "Biaxial Molecular Arrangement of Rod-Disc Molecule under an

\title{
Electric Field"
}

\author{
Kwang-Un Jeong, ${ }^{1,2}$ Alexander J. Jing, ${ }^{1}$ Bart Mansdorf, ${ }^{1}$ Matthew J. Graham, ${ }^{1}$ \\ Dengke Yang, ${ }^{3}$ Frank W. Harris ${ }^{1}$ and Stephen Z. D. Cheng $*^{1}$
}

${ }^{1}$ Maurice Morton Institute and Department of Polymer Science, The University of Akron, Akron, Ohio 44325-3909, ${ }^{2}$ School of Advanced Materials Engineering, Chonbuk National University, Jeonju, 561-756, Korea, ${ }^{3}$ Liquid Crystal Institute, Kent State University, Kent Ohio 44242

* To whom the correspondence should be addressed. E-mail: scheng@uakron.edu

\section{Experimental Section}

Materials and sample preparation. A rod-disc combined LC (RDn, here $\mathrm{n}$ is the number of carbon atoms between the rod and the disc mesogens, $n=12$ ) was synthesized via a four-step condensation reaction. ${ }^{1}$ The molecular weight of RD12 was $2490 \mathrm{~g} / \mathrm{mol}$. The compound was purified by silica gel chromatography using chloroform/hexane (6/1) mixed solvent as an eluent, and then, vacuum-dried. The purity and chemical structure of the compound was verified by thin-layer chromatography, MALDI-TOF mass spectrometry, elemental analysis, and nuclear magnetic resonance spectroscopy.

The purified compound was kept in a vacuum before characterization. For DSC experiments, the sample weight was about $2.0 \mathrm{mg}$ and the pan weights were kept constant with a precision of $\pm 0.001 \mathrm{mg}$. In order to obtain detailed structure information via 2D WAXD experiments, the RD12 sample was oriented under the DC electrical field. To the investigate the effect of the electric field (ED) on the RD12 orientation at different direct current (DC) voltages by PLM, the RD12 sample was placed in between the top and bottom ITO-coated conducting 
glasses to construct electro-optic LC cells. The film thickness in the cell was also controlled to be $10 \mu \mathrm{m}$ by spacers.

Equipment and Experiments. The thermal transitions were studied using a Perkin-Elmer PYRIS Diamond DSC with an Intracooler 2P apparatus. The temperatures and heat flows were calibrated using material standards (benzoic acid and indium) at a $2.5^{\circ} \mathrm{C} / \mathrm{min}$ cooling and subsequent heating rate. The heating experiments always preceded the cooling experiments in order to eliminate previous thermal histories, and the cooling and heating rates were kept identical. The transition temperatures were determined by measuring the onset and peak temperatures from both the cooling and heating scans at different rates.

2D WAXD experiments on the oriented samples were conducted using an imaging system (Rigaku, R-AXIS-IV) with an $18 \mathrm{~kW}$ rotating anode X-ray generator. The 2D WAXD patterns were taken from the several places of the specimen. The diameter of X-ray beam we used is around the $0.3 \mathrm{~mm}$. A $30 \mathrm{~min}$ exposure time was required for a high-quality pattern. The background scatterings were subtracted from the sample scans.

Morphological observations on the micrometer scale were conducted using polarized light microscopy (PLM, Olympus BH-2) coupled with a Mettler heating stage (FP-90). The temperature of this hot stage was calibrated to be within $\pm 0.5^{\circ} \mathrm{C}$.

The Cerius $^{2}$ (Version 4.6) simulation software from Accelrys was used to calculate the energy minimized geometry of the RD12 molecular disc in the isolated gas-phase utilizing the COMPASS force field. To apply a DC electric field on the electro-optic LC cells, the ITO layers were connected to a DC voltage generator (PZD350, Trek).

\section{Reference:}


1. Jeong, K.-U.; Jing, A. J.; Monsdorf, B.; Graham, M. J.; Harris, F. W.; Cheng, S. Z. D. J. Phys. Chem. B 2007, 111, 767. 